\title{
Weighted Stress Function Method for Multiobjective Evolutionary Algorithm Based on Decomposition
}

\author{
Roman Denysiuk and António Gaspar-Cunha \\ Department of Polymer Engineering, University of Minho, Campus de Azurém \\ 4800-058, Guimarães, Portugal \\ \{roman; agc@dep.uminho.pt\}
}

\begin{abstract}
Multiobjective evolutionary algorithm based on decomposition (MOEA/D) is a well established state-of-the-art framework. Major concerns that must be addressed when applying MOEA/D are the choice of an appropriate scalarizing function and setting the values of main control parameters. This study suggests a weighted stress function method (WSFM) for fitness assignment in MOEA/D. WSFM establishes analogy between the stress-strain behavior of thermoplastic vulcanizates and scalarization of a multiobjective optimization problem. The experimental results suggest that the proposed approach is able to provide a faster convergence and a better performance of final approximation sets with respect to quality indicators when compared with traditional methods. The validity of the proposed approach is also demonstrated on engineering problems.
\end{abstract}

\section{Introduction}

This study considers a multiobjective optimization problem (MOP) of the form

$$
\begin{array}{rll}
\operatorname{minimize} & \left(f_{1}(\boldsymbol{x}), \ldots, f_{m}(\boldsymbol{x})\right) \\
\text { subject to } & c_{i}(\boldsymbol{x}) \leq 0 & i=1, \ldots, k \\
& l_{j} \leq x_{j} \leq u_{j} \quad j=1, \ldots, n
\end{array}
$$

where $\boldsymbol{x}=\left(x_{i}, \ldots, x_{n}\right)$ is the decision vector, $\boldsymbol{f}=\left(f_{1}, \ldots, f_{m}\right)$ is the objective vector, $\boldsymbol{c}=\left(c_{1}, \ldots, c_{k}\right)$ is the vector of inequality constraints, $l_{j}$ and $u_{j}$ are the lower and upper bounds of the $j$-th variable, respectively.

When solving a MOP, multiobjective evolutionary algorithms (MOEAs) attempt to approximated the Pareto set that rises from the conflicting nature of the involved objectives. In this process, MOEAs rely on three major mechanisms such as selection, variation and replacement. Most variation operators are adopted from single objective optimization by inserting existing mechanisms for producing offspring into a framework able to deal with multiple objectives. Parents selection and replacement are based on some fitness assignment scheme intended to emphasize and propagate promising individuals in the presence of multiple objectives. The convergence and diversity are two essential issues that 
must be addressed by the fitness assignment. The convergence refers to guiding the population towards the Pareto set. The diversity implies that a diverse set of solutions is maintained. In current state-of-the-art MOEAs, there can be distinguished three major approaches to fitness assignment.

A dominance-based strategy probably is the most frequently used one. It relies on the concept of the Pareto dominance and is usually combined with some diversity preserving mechanism [2]. Its advantage is related to the correspondence with the concept of optimality in multiobjective optimization. Though the performance of such mechanism severely deteriorates when the number of objectives is increased. Also, because the diversity is usually considered as a second sorting criterion, dominance-based MOEAs may face substantial difficulties in solving MOPs due to severe loss of diversity.

Indicator-based MOEAs employ quality indicators to assign fitness to individuals in the population [19]. The development of such MOEAs is based on the idea that it can be beneficial to explicitly optimize a measure that is used for algorithms comparison. Moreover, some quality indicators possess good theoretical properties and are Pareto compliant. The difficulty in their application arises from a high computational cost. In particular, a computational time of the hypervolume grows exponentially with the number of objectives. This significantly limits its applicability. Approximating the hypervolume requires trade-off between accuracy and complexity. The indicator based fitness assignment can also face difficulties in balancing the convergence and diversity.

Decomposition-based approaches aim at decomposing a MOP into a number of subproblems and solving them simultaneously. The decomposition can be based on the aggregation of multiple objectives into a scalarizing function using traditional mathematical techniques [14]. In such MOEAs, convergence is achieve by optimizing the corresponding scalarizing function whereas diversity is ensured by a well distributed set of weight vectors. MOEAs relying on scalarization usually works well on problems with a large number of objectives. The major advantage is their efficiency. Other MOEAs use directional vectors for associating individuals with the corresponding direction so that the diversity of population members is ensured [12] [13]. Decomposition-based approaches typically require a set of weights or directional vectors being provided in advance. Generating such set often is not an easy task, especially when the number of dimensions is high. Alternatively, the decomposition can be performed by generating a grid using polar coordinates [5] or by exploring the angles between population members in the objective space [4].

MOEA/D is a representative state-of-the-art approach relying on decomposition by means of scalarization. MOEA/D associates each population member with a subproblem defined by a scalarizing function. In the end, individuals represent solutions to the corresponding subproblems. Neighborhood relations among subproblems are defined using the distances between the weight vectors. These relations are exploited during the search. Since its advent, MOEA/D has been increasingly investigated. This resulted in a number of different variants, including studies of different reproduction operators and improvements of its 
core framework. The effect of different scalarizing functions was studied in [7]. The results of this study suggest that a proper choice of scalarizing function is an important issue for the performance of MOEA/D. Another issue that heavily influences the search ability of MOEA/D is setting the values of control parameters. Mechanisms involving adaptation of control parameters during the search appeared effective in enhancing the performance of MOEA/D. Recently, it was shown that a better exploration of the search space can be achieved when performing replacement in the neighborhood of the subproblem that best matches offspring [16]. Another important issue in MOEA/D is an efficient allocation of computational resources between different subproblems [18].

This study focuses on the MOEA/D fitness assignment mechanism. A new scalarizing function is suggested to guide the search, which is called a weighted stress function method (WSFM). The use of WSFM is motivated by its promising behavior as a preference articulation method [6]. The inspiration for WSFM stems from mechanics, namely from the stress-strain behavior of thermoplastic vulcanizates [1]. WSFM has particular characteristics that are different from traditional methods for scalarization. As experimental results suggest, MOEA/D with WSFM can provide better results when compared with state-of-the-art scalarization methods.

The remainder of this paper is organized as follows. Section 2 describes the MOEA/D framework. Section 3 introduces the WSFM for MOEA/D. Section 4 discusses the results of the experimental study. Finally, Section 5 presents conclusions of the study and outlines some possible future work.

\section{Multiobjective Evolutionary Algorithm Based on Decomposition}

\subsection{Algorithm}

The interest from research community and effort in improving the performance led to several variants of MOEA/D. As this study focuses on the fitness assignment, MOEA/D is considered with two different replacement variants. The first uses the mating pool. The second selects the most suitable subproblem to offspring and uses its neighborhood. In the following, this two variants are referred using notation from corresponding papers as MOEA/D [11] [17] and MOEA/DGR [16], respectively. The outline of MOEA/D is given as follows.

Input:

- $\delta$ - probability for mating pool;

- $T$ - neighborhood size;

- $n_{r}$ - maximum number of individuals replaced by offspring;

- $\mu$ - population size;

- maxGen - maximum number of generations.

\section{Output:}

- $\left\{\boldsymbol{x}^{1}, \ldots, \boldsymbol{x}^{\mu}\right\}$ - approximation to the Pareto set; 
- $\left\{\boldsymbol{f}\left(\boldsymbol{x}^{1}\right), \ldots, \boldsymbol{f}\left(\boldsymbol{x}^{\mu}\right)\right\}$ - approximation to the Pareto front.

\section{Step 1 Initialization}

Step 1.1 Read input parameters;

Step 1.2 Generate a set of weight vectors $W=\left\{\boldsymbol{w}^{1}, \ldots, \boldsymbol{w}^{\mu}\right\}$, where $\sum_{j=1}^{m} w_{j}^{i}=$ $1, \forall i \in\{1, \ldots, \mu\}$;

Step 1.3 For each weight vector, select the $T$ closest weight vectors by:

1. Computing the Euclidean distance between any two weight vectors;

2. Setting $B(i)=\left\{i_{1}, \ldots, i_{T}\right\}, \forall i \in\{1, \ldots, \mu\}$, where $\boldsymbol{w}^{i_{1}}, \ldots, \boldsymbol{w}^{i_{T}}$ are the $T$ closest weight vectors to $\boldsymbol{w}^{i}$;

Step 1.4 Randomly generate an initial population. Evaluate the population; Step 1.4 Initialize a reference point, $\boldsymbol{z}$, by setting $z_{j}=\min f_{j}\left(\boldsymbol{x}^{i}\right) \forall i \in$ $\{1, \ldots, \mu\}, \forall j \in\{1, \ldots, m\}$.

\section{Step 2 Evolution}

For each $i=1, \ldots \mu$ do:

\section{Step 2.1 Selection}

1. Select mating pool:

$$
P_{m}= \begin{cases}B(i) & \text { with probability } \delta \\ \{1, \ldots, \mu\} & \text { otherwise }\end{cases}
$$

2. From $P_{m}$, select parents for reproduction;

\section{Step 2.2 Variation}

1. Apply evolutionary operators on parents to produce offspring $\boldsymbol{y}$;

2. Evaluate offspring.

\section{Step 2.3 Update}

For each $j=1, \ldots, m: z_{j}=f_{j}(\boldsymbol{y})$ if $f_{j}(\boldsymbol{y})<z_{j}$.

\section{Step 2.4 Replacement}

Select pool for replacement $P_{r}$, set $c=0$ and do the following:

1. If $c=n_{r}$ or $P_{r}=\emptyset$, go to Step 3. Otherwise, pick an index $j$ from $P_{r}$;

2. If fitness $\left(\boldsymbol{f}(\boldsymbol{y}) \mid \boldsymbol{w}^{j}\right)<$ fitness $\left(\boldsymbol{f}\left(\boldsymbol{x}^{j}\right) \mid \boldsymbol{w}^{j}\right)$, then set $\boldsymbol{x}^{j}=\boldsymbol{y}$ and $c=c+1$;

3. Remove $j$ from $P_{r}$ and got to 1 .

Step 3 If the stopping criterion is not met, go to Step 2. Otherwise, return Output.

Depending on the parameter settings, the above depicted algorithm defines one of the three MOEA/D variants considered in the present study. These were originally presented in [11] [16] and [17]. 


\subsection{Scalarizing Functions}

MOEA/D decomposes a MOP into a set of single-objective subproblems by means of scalarization. Scalarization relies on a scalarizing function to compute a scalar value for the given objectives and weights. The choice of a scalarizing function is an important issue that greatly influences the performance of MOEA/D. Most scalarizing functions are adopted from traditional programming methods for solving MOPs [14]. Owing to the concern of the present study, frequently used scalarizing functions are briefly reviewed in the following.

Weighted Sum (WSUM)

The weighted sum method associates each objective with a weight and minimizes the weighted sum of the objectives. The scalarizing function can be defined as

$$
\text { minimize } g(\boldsymbol{f} \mid \boldsymbol{w})=\sum_{i=1}^{m} w_{i} f_{i} .
$$

The advantages of this method are that it does not need a reference point and the resulting scalar optimization problem is convex. The major shortcoming of this method is that it fails to find solutions in nonconvex regions of the Pareto front.

\section{Chebyshev (CHB)}

For a reference point $\boldsymbol{z}=\left(z_{1}, \ldots, z_{m}\right)$, the scalarizing function based on Chebyshev method can be defined as

$$
\text { minimize } g(\boldsymbol{f} \mid \boldsymbol{w})=\max _{1 \leq i \leq m} w_{i}\left|f_{i}-z_{i}\right|
$$

This method belongs to the group of weighted metric methods that seek to minimize the distance between some reference point and the feasible objective region where the weighted $L_{p}$ metric is used for measuring this distance. The problem in (3) is obtained for $p=\infty$. This method can find solutions in convex and nonconvex regions of the Pareto front. The drawbacks are that it cannot distinguish weakly Pareto optimal solutions and does not provide a uniform distribution of solutions along the Pareto front.

Penalty Boundary Intersection (PBI)

The penalty boundary intersection method was suggested in [17] in order to generate a more uniform approximation to the Pareto front by MOEA/D. The scalarizing function is given by

$$
\text { minimize } g(\boldsymbol{f} \mid \boldsymbol{w})=d_{1}+\theta d_{2}
$$

where

$$
\begin{aligned}
d_{1} & =\frac{\left\|(\boldsymbol{f}-\boldsymbol{z})^{\mathrm{T}} \boldsymbol{w}\right\|}{\|\boldsymbol{w}\|} \\
d_{2} & =\left\|\boldsymbol{f}-\left(\boldsymbol{z}+d_{1} \frac{\boldsymbol{w}}{\|\boldsymbol{w}\|}\right)\right\| .
\end{aligned}
$$

The major advantage of this method is that it can provide a reasonably uniform distribution of solutions along the Pareto front. Though it comes at the cost of specifying the value of $\theta$. Different settings of this parameter can heavily affect the performance of MOEA/D. 


\subsection{Constraint Handling}

Real-world problems often involve constraints that must be satisfied. This study considers the constraint handling technique for MOEA/D presented in [9], as it proved effective on a set of challenging constrained problems. This method relies on a penalty function, $p$, that is dynamically adjusted. For a given individual, the degree of constraint violation, $C V(\boldsymbol{x})$, is estimated as

$$
C V(\boldsymbol{x})=\sum_{j=1}^{k} \max \left(c_{j}(\boldsymbol{x}), 0\right) .
$$

The value of $p$ is computed on the basis of $C V$ as

$$
p(\boldsymbol{x})= \begin{cases}s_{1} C V(\boldsymbol{x})^{2} & \text { if } C V(\boldsymbol{x})<\tau \\ s_{1} \tau^{2}+s_{2}(C V(\boldsymbol{x})-\tau), & \text { otherwise }\end{cases}
$$

A threshold value, $\tau$, is defined as

$$
\tau=C V_{\min }+0.3\left(C V_{\max }-C V_{\min }\right)
$$

where $s_{1}=0.01$ and $s_{2}=20$ are scaling parameters, $C V_{\min }$ and $C V_{\max }$ are the minimum and maximum values of constraint violation in the current population. The penalty function encourages the exploration of both feasible and infeasible regions. The role of parameter $\tau$ is to control the amount of penalty. Thus, the fitness of the $i$-th population member is given as

$$
\text { fitness }(\boldsymbol{x} \mid \boldsymbol{w})=g(\boldsymbol{f}(\boldsymbol{x}) \mid \boldsymbol{w})+p(\boldsymbol{x}) .
$$

\section{$3 \quad$ Weighted Stress Function Method}

\subsection{Analogy with Rubber Elasticity}

The weighted stress function method (WSFM) is inspired by the stress-strain behavior of thermoplastic vulcanizates (TPVs) [1]. These materials are a particular group of thermoplastic elastomers possessing high performance elastic and mechanical properties. Stress and strain are two different but closely related concepts. Stress is defined as force per unit area that can cause a change in an object or a physical body. Strain is defined as the amount of deformation experienced due to the application of stress. The relationship between the stress and strain that a particular material exhibits is displayed by the stress-strain curve.

A typical structure of a TPV consists of a very high volume fraction $(0.40<$ $\left.v_{p}<0.9\right)$ of fully cured elastomeric particles surrounded by a continuous thermoplastic matrix. The majority of experimental studies on this type of material show that the elasticity of the material increases when the volume fraction of the elastomeric particle is increased from 0.0 to 1.0. Figure 1 illustrates the

stress-strain behavior for different values of $v_{p}$. This figure shows that there are 


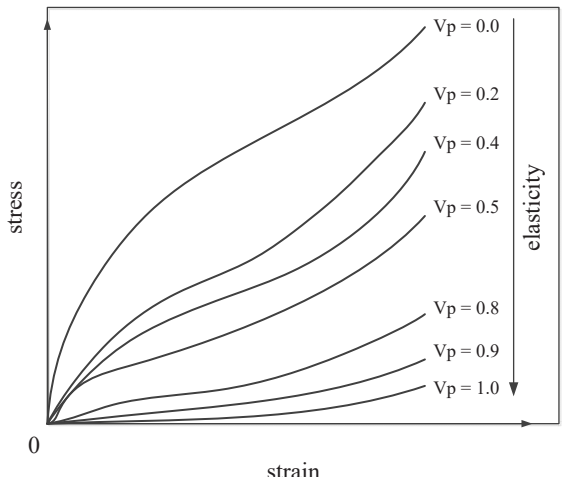

Fig. 1: Stress strain behavior.

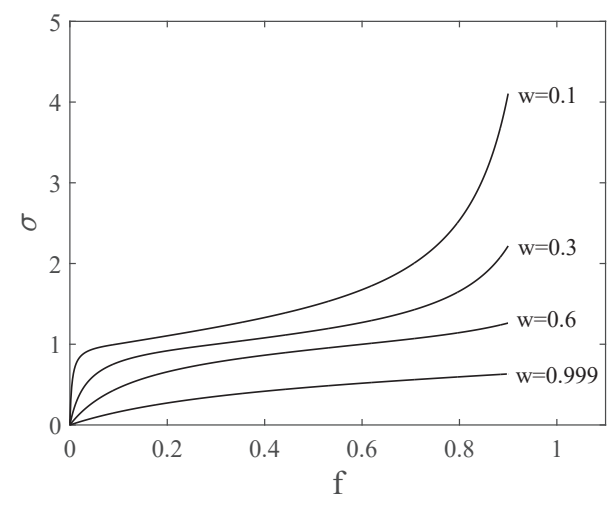

Fig. 2: Stress values for different weights

zones with different rates of stress and strain, with those having a nonlinear relationship.

WSFM establishes an analogy between the stress-strain behavior of TPV materials and a stress function that takes into account weights defined for each objective. This method assumes that the difference between the ideal point and each solution induces a stress on that solution. The stress depends on the weights associated with the objectives of the given solution. Similarly to the values of $v_{p}$, the weights range in the interval $[0,1]$ and resembles the role that $v_{p}$ plays in increasing or decreasing the stress.

\subsection{Scalarizing Function}

WSFM transforms each objectives, $f_{i}$, into a stress, $\sigma_{i}$, depending on the value of associated weight, $w_{i}$. The WSFM problem seeks to minimize the largest stress associated with the given solutions. The scalarizing problem is of the form

$$
\operatorname{minimize} g(\boldsymbol{f} \mid \boldsymbol{w})=\max _{1 \leq i \leq m} \sigma_{i}\left(f_{i}, w_{i}\right) .
$$

The calculation of the stresses requires the normalization of the objective values so that they are in the range $[0,1]$. This is done as

$$
f_{i}=\frac{f_{i}-f_{i}^{\min }}{f_{i}^{\max }-f_{i}^{\min }}
$$

where $f_{i}^{\min }$ and $f_{i}^{\max }$ are the minimum and maximum values of the $i$-th objective in the current population.

Assuming minimization of the $m$ objectives $\left(f_{1}, \ldots, f_{m}\right)$, for the weight vector $\left(w_{1}, \ldots, w_{m}\right)$, the stress $\sigma_{i}$ associated with the $i$-th objective is calculated as

$$
\sigma_{i}\left(f_{i}, w_{i}\right)=\left(1+\omega_{i}\left(f_{i}, w_{i}\right)\right) \xi_{i}\left(w_{i}\right)
$$


where

$$
\begin{gathered}
\omega_{i}\left(f_{i}, w_{i}\right)=\left\{\begin{array}{l}
\frac{\tan \left(\frac{\pi}{\psi_{i}\left(w_{i}\right)}\left(f_{i}-w_{i}\right)\right)}{\tan \left(\frac{\pi}{\phi_{i}\left(w_{i}\right)} w_{i}-\delta_{1}\right)} \frac{\psi_{i}\left(w_{i}\right)}{\phi_{i}\left(w_{i}\right)}, f_{i} \geq w_{i} \\
\frac{\tan \left(\frac{\pi}{\phi_{i}\left(w_{i}\right)}\left(f_{i}-w_{i}\right)\right)}{\tan \left(\frac{\pi}{\phi_{i}\left(w_{i}\right)} w_{i}\right)}, \quad f_{i}<w_{i}
\end{array}\right. \\
\psi_{i}\left(w_{i}\right)=\frac{3}{4} w_{i}^{2}+2\left(1-w_{i}\right)+\delta_{1} \\
\phi_{i}\left(w_{i}\right)=\frac{3}{4} w_{i}^{2}+2 w_{i}+\delta_{1} \\
\xi_{i}\left(w_{i}\right)=1-\frac{\tan \left(\frac{\pi}{2\left(1+\delta_{2}\right)}\left(2 w_{i}-1\right)\right)}{\tan \left(\frac{\pi}{2\left(1+\delta_{2}\right)}\right)} .
\end{gathered}
$$

To ensure that $\sigma_{i}$ does not assume infinite values, the extreme values of weights are projected as

$$
w_{i}=\min \left(\max \left(w_{i}, \epsilon\right), 1-\epsilon\right) .
$$

Figure 2 plots stresses for different weight values. High values of weights correspond to lower stresses. Also, it can be seen that there is a nonlinear relationship.

Figure 3 illustrates contour lines for the different scalarizing functions referred above. In the plots, dashed lines show the directions of search. For CHB and WSFM, these lines correspond to the cases when the terms in the max function in (3) and (12) are equal. For bold dashed lines, the contour lines are depicted by bold solid lines. These lines divide the objective space into region with solutions located under these line being better than those in the other region. The contour line for WSFM is a line that is perpendicular to the direction of search. For the CHB approach, this is a polygonal line with the right angle. A polygonal line is also the contour line for PBI, though the angle depends on the value of $\theta$. A unique characteristic of WSFM can be identified from the corresponding plot. Although the shape of contour lines is identical to CHB, in WSFM there are nonlinear lines that define the directions of search.

\section{Computational Experiments}

This section discusses the computational experiments carried out to investigate the performance of MOEA/D when using different scalarizing functions for fitness assignment. The experiments are divided into three different parts according to employed test problems and MOEA/D variants. These include state-of-theart test suites, problems with complicated Pareto sets and engineering design problems. For each problem, 30 independent runs of each MOEA/D variant are performed. The results are quantitatively assessed using the epsilon and hypervolume indicators, which are Pareto compliant quality indicators [10]. 

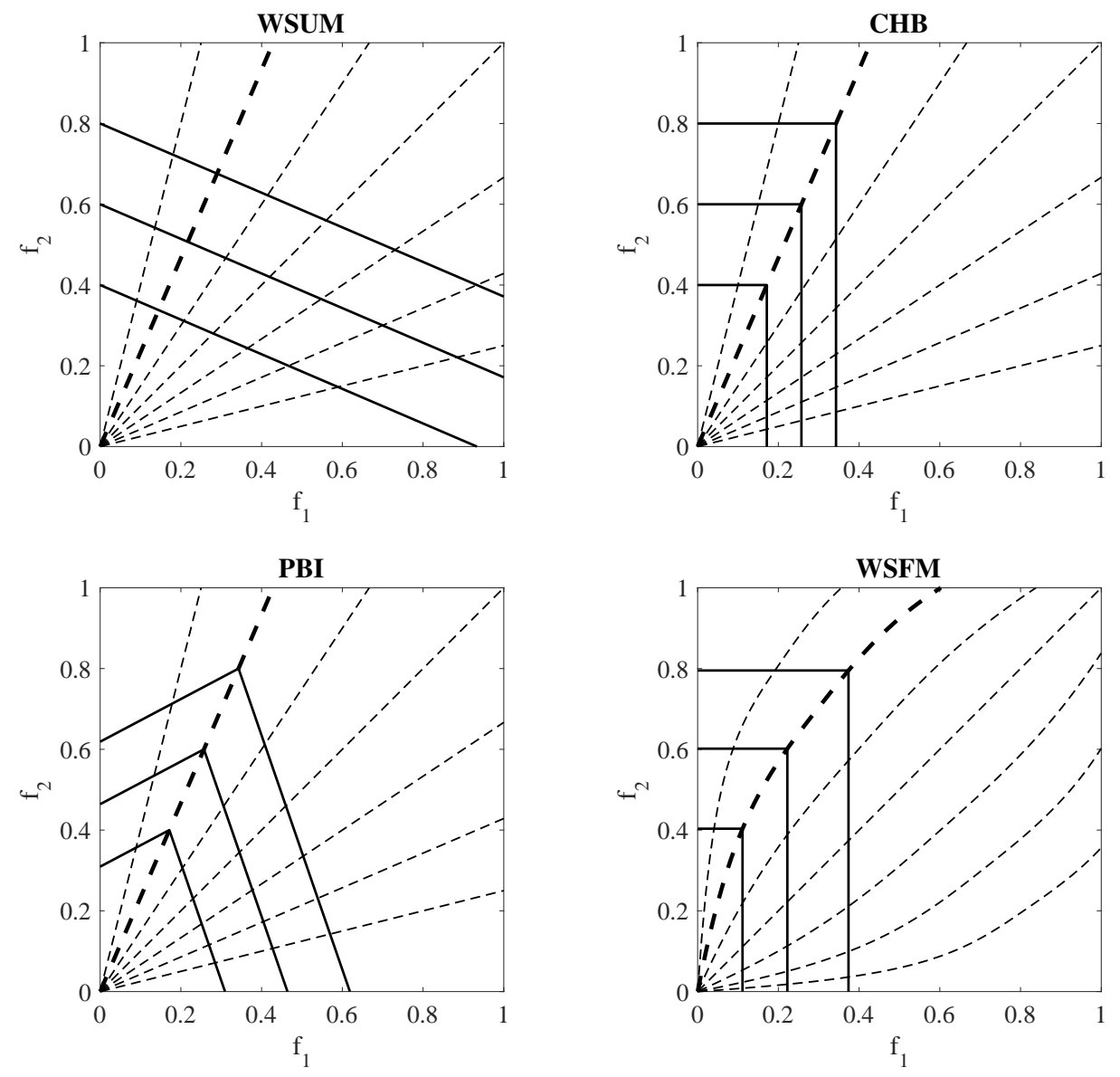

Fig. 3: Contour lines for different scalarizing functions.

\subsection{ZDT and DTLZ Problems}

Problems from the ZDT and DTLZ suites are widely used for benchmarking MOEAs. Similarly to the study [17] introducing the MOEA/D framework, this study performs experiments adopting continuous ZDT and three-objective DTLZ1 and DTLZ2 problems. These experiments aim to test a genetic algorithm variant of MOEA/D-WSFM, which uses the SBX crossover and polynomial mutation for reproduction. MOEA/D is run for 300 generations with $\mu=100$. The other control parameters are $T=n_{r}=10$ and $\delta=1$.

Table 1 summarizes the results with respect to the quality indicators. It is evident that MOEA/D-WSFM is the best performing variant. For the two replacement strategies, WSFM is only outperformed by CHB on the ZDT3 problem, 
Table 1: Results for ZDT and DTLZ problems. The values refer to the median and interquartile range of the epsilon (eps) and hypervolume (hv) indicators.

\begin{tabular}{|c|c|c|c|c|}
\hline & M & $\mathrm{CHB}$ & PBI & M \\
\hline \multicolumn{5}{|c|}{ MOEA/D } \\
\hline \multirow{2}{*}{ ZDT1 } & eps 2.90e-02 (2.3e-05) & $8.20 \mathrm{e}-03(4.7 \mathrm{e}-05)$ & $3.43 \mathrm{e}-01(3.7 \mathrm{e}-02)$ & 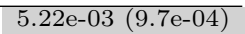 \\
\hline & hv $8.62 \mathrm{e}-01(1.1 \mathrm{e}-06)$ & $8.72 \mathrm{e}-01(2.8 \mathrm{e}-05)$ & $7.08 \mathrm{e}-01(2.3 \mathrm{e}-02)$ & $8.72 \mathrm{e}-01(4.9 \mathrm{e}-05)$ \\
\hline \multirow{2}{*}{ ZDT2 } & eps $3.82 \mathrm{e}-01(0.0 \mathrm{e}+00)$ & $6.31 \mathrm{e}-03(5.0 \mathrm{e}-05)$ & $9.98 \mathrm{e}-01(2.3 \mathrm{e}-03)$ & $5.36 \mathrm{e}-03(3.4 \mathrm{e}-05)$ \\
\hline & hv $2.10 \mathrm{e}-01(0.0 \mathrm{e}+00)$ & $5.37 \mathrm{e}-01(2.8 \mathrm{e}-05)$ & $1.12 \mathrm{e}-01(2.4 \mathrm{e}-03)$ & $5.39 \mathrm{e}-01(4.2 \mathrm{e}-04)$ \\
\hline \multirow{2}{*}{ ZDT3 } & eps 2.65e-01 (1.2e-02) & $1.72 \mathrm{e}-02(5.0 \mathrm{e}-05)$ & $2.94 \mathrm{e}-01(8.2 \mathrm{e}-02)$ & $1.78 \mathrm{e}-01(2.0 \mathrm{e}-01)$ \\
\hline & hv $5.56 \mathrm{e}-01(2.2 \mathrm{e}-02)$ & $7.24 \mathrm{e}-01(4.4 \mathrm{e}-05)$ & $6.09 \mathrm{e}-01(6.3 \mathrm{e}-02)$ & $7.04 \mathrm{e}-01(4.1 \mathrm{e}-02)$ \\
\hline \multirow{2}{*}{ ZDT4 } & eps $3.15 \mathrm{e}-02(1.1 \mathrm{e}-02)$ & $1.31 \mathrm{e}-02(4.1 \mathrm{e}-03)$ & $3.33 \mathrm{e}-01(4.3 \mathrm{e}-02)$ & $1.23 \mathrm{e}-02(1.7 \mathrm{e}-02)$ \\
\hline & hv $8.60 \mathrm{e}-01(2.5 \mathrm{e}-03)$ & $8.65 \mathrm{e}-01(5.9 \mathrm{e}-03)$ & $7.09 \mathrm{e}-01(2.8 \mathrm{e}-02)$ & $8.65 \mathrm{e}-01(6.4 \mathrm{e}-03)$ \\
\hline \multirow{2}{*}{ ZDT6 } & eps $4.31 \mathrm{e}-01$ (2.5e-08) & $03(1.4 \mathrm{e}-05)$ & $1.13 \mathrm{e}-01(3.9 \mathrm{e}-05)$ & $5.23 \mathrm{e}-03(2.0 \mathrm{e}-05)$ \\
\hline & hv $2.10 \mathrm{e}-01(1.9 \mathrm{e}-08)$ & $01(3.6 \mathrm{e}-06)$ & $5.92 \mathrm{e}-01(5.6 \mathrm{e}-06)$ & $6.11 \mathrm{e}-01(7.7 \mathrm{e}-07)$ \\
\hline \multirow{2}{*}{ DTLZ1 } & eps 6.29e-01 (3.7e-02) & $9.61 \mathrm{e}-02(2.0 \mathrm{e}-03)$ & $2.17 \mathrm{e}-01(1.0 \mathrm{e}-01)$ & $6.29 \mathrm{e}-02(2.1 \mathrm{e}-03)$ \\
\hline & hv $3.51 \mathrm{e}-01(1.8 \mathrm{e}-01)$ & $1.06 \mathrm{e}+00(3.8 \mathrm{e}-03)$ & $8.33 \mathrm{e}-01(6.4 \mathrm{e}-02)$ & $1.12 \mathrm{e}+00(4.3 \mathrm{e}-03)$ \\
\hline \multirow{2}{*}{ DTLZ2 } & eps $4.22 \mathrm{e}-01(3.3 \mathrm{e}-08)$ & $9.83 \mathrm{e}-02(5.1 \mathrm{e}-04)$ & $7.57 \mathrm{e}-02(1.4 \mathrm{e}-03)$ & $5.57 \mathrm{e}-02(2.9 \mathrm{e}-04)$ \\
\hline & hv $0.00 \mathrm{e}+00(0.0 \mathrm{e}+00)$ & $6.69 \mathrm{e}-01(4.4 \mathrm{e}-03)$ & $7.41 \mathrm{e}-01(5.6 \mathrm{e}-05)$ & $7.46 \mathrm{e}-01(2.7 \mathrm{e}-04)$ \\
\hline \multicolumn{5}{|c|}{ MOEA/D-GR } \\
\hline \multirow{2}{*}{ ZDT1 } & $-01(3.06-01)$ & & $01-2.1$ & $.220-00(0.46$ \\
\hline & hv $2.15 \mathrm{e}-01$ (9.0e-02) & $8.72 \mathrm{e}-01(6.1 \mathrm{e}-05)$ & $7.08 \mathrm{e}-01(1.4 \mathrm{e}-$ & $8.72 \mathrm{e}-01(3.6 \mathrm{e}-05)$ \\
\hline \multirow{2}{*}{ ZDT2 } & eps $1.00 \mathrm{e}+00(0.0 \mathrm{e}+00)$ & $6.35 \mathrm{e}-03(1.3 \mathrm{e}-04)$ & $9.98 \mathrm{e}-01(1.7 \mathrm{e}-02)$ & $5.37 \mathrm{e}-03(3.3 \mathrm{e}-05)$ \\
\hline & hv $1.10 \mathrm{e}-01(0.0 \mathrm{e}+00)$ & $5.37 \mathrm{e}-01(1.3 \mathrm{e}-04)$ & $1.12 \mathrm{e}-01(1.7 \mathrm{e}-02)$ & $5.38 \mathrm{e}-01(3.7 \mathrm{e}-05)$ \\
\hline \multirow{2}{*}{ ZDT3 } & eps 5.03e-01 (7.6e-03) & $1.72 \mathrm{e}-02(5.1 \mathrm{e}-05)$ & $2.93 \mathrm{e}-01(8.9 \mathrm{e}-02)$ & $1.78 \mathrm{e}-01(1.7 \mathrm{e}-01)$ \\
\hline & hv $1.42 \mathrm{e}-01(1.1 \mathrm{e}-01)$ & $7.24 \mathrm{e}-01(3.3 \mathrm{e}-05)$ & $6.16 \mathrm{e}-01(6.0 \mathrm{e}-02)$ & $7.04 \mathrm{e}-01(2.1 \mathrm{e}-02)$ \\
\hline \multirow[b]{2}{*}{ ZDT4 } & eps $2.12 \mathrm{e}+00(8.0 \mathrm{e}-01)$ & $1.22 \mathrm{e}-02(3.8 \mathrm{e}-03)$ & $3.48 \mathrm{e}-01(4.1 \mathrm{e}-02)$ & $1.06 \mathrm{e}-02(3.6 \mathrm{e}-03)$ \\
\hline & hv $0.00 \mathrm{e}+00(0.0 \mathrm{e}+00)$ & $8.65 \mathrm{e}-01(4.0 \mathrm{e}-03)$ & $7.01 \mathrm{e}-01(2.7 \mathrm{e}-02)$ & $8.67 \mathrm{e}-01(2.9 \mathrm{e}-03)$ \\
\hline \multirow{2}{*}{ ZDT6 } & eps $4.31 \mathrm{e}-01(1.1 \mathrm{e}-05)$ & $6.07 \mathrm{e}-03(8.6 \mathrm{e}-06)$ & $1.13 \mathrm{e}-01(4.6 \mathrm{e}-05)$ & $5.23 \mathrm{e}-03(1.6 \mathrm{e}-05)$ \\
\hline & hv $2.10 \mathrm{e}-01(8.5 \mathrm{e}-06)$ & $6.11 \mathrm{e}-01(5.3 \mathrm{e}-06)$ & $5.83 \mathrm{e}-01(2.6 \mathrm{e}-05)$ & $6.11 \mathrm{e}-01(8.0 \mathrm{e}-06)$ \\
\hline \multirow{2}{*}{ DTLZ1 } & eps $6.76 \mathrm{e}-01(9.4 \mathrm{e}-01)$ & $9.71 \mathrm{e}-02(2.4 \mathrm{e}-03)$ & $1.60 \mathrm{e}-01(9.8 \mathrm{e}-02)$ & $6.13 \mathrm{e}-02(4.0 \mathrm{e}-03)$ \\
\hline & hv $0.00 \mathrm{e}+00(2.7 \mathrm{e}-01)$ & $1.06 \mathrm{e}+00(4.0 \mathrm{e}-03)$ & $8.83 \mathrm{e}-01(7.0 \mathrm{e}-02)$ & $1.12 \mathrm{e}+00(3.3 \mathrm{e}-03)$ \\
\hline \multirow{2}{*}{ DTLZ2 } & eps $4.04 \mathrm{e}-01(5.2 \mathrm{e}-02)$ & $9.85 \mathrm{e}-02(4.7 \mathrm{e}-04)$ & $8.65 \mathrm{e}-02(7.7 \mathrm{e}-04)$ & $5.56 \mathrm{e}-02(6.2 \mathrm{e}-04)$ \\
\hline & hv $3.44 \mathrm{e}-02(7.8 \mathrm{e}-02)$ & $6.68 \mathrm{e}-01(2.6 \mathrm{e}-03)$ & $7.38 \mathrm{e}-01(2.5 \mathrm{e}-04)$ & $7.46 \mathrm{e}-01(3.0 \mathrm{e}-04)$ \\
\hline
\end{tabular}

whose Pareto front consists of five disconnected parts. This suggests that WSFM works better for continuous than disconnected shapes of the Pareto front.

Figure 4 depicts the result with the best hypervolume obtained by WSFM for two- and three-objective problems having distinct characteristics. The presented plots show that adequate approximations can be obtained for different Pareto front geometries. Although WSFM loses to CHB on ZDT3 regarding the quality indicators, the Pareto front of this problem is adequately approximated as well.

\subsection{Problems with Complicated Pareto Sets}

Problems with complicated Pareto sets were introduced in [11] specifically for investigating advantages of MOEA/D. They were designed to resemble properties of real-world problems, such as complex nonlinear interactions between the decision variables. Several studies showed that decomposition based approaches are especially useful for handling such problems. The present study tests a differential evolution variant of MOEA/D-WSFM and its ability to deal with challenging characteristics of these problems. MOEA/D is run for 500 generations with $\mu=300$. The other parameter settings are $\delta=0.9, T=20$ and $n_{r}=2$. 

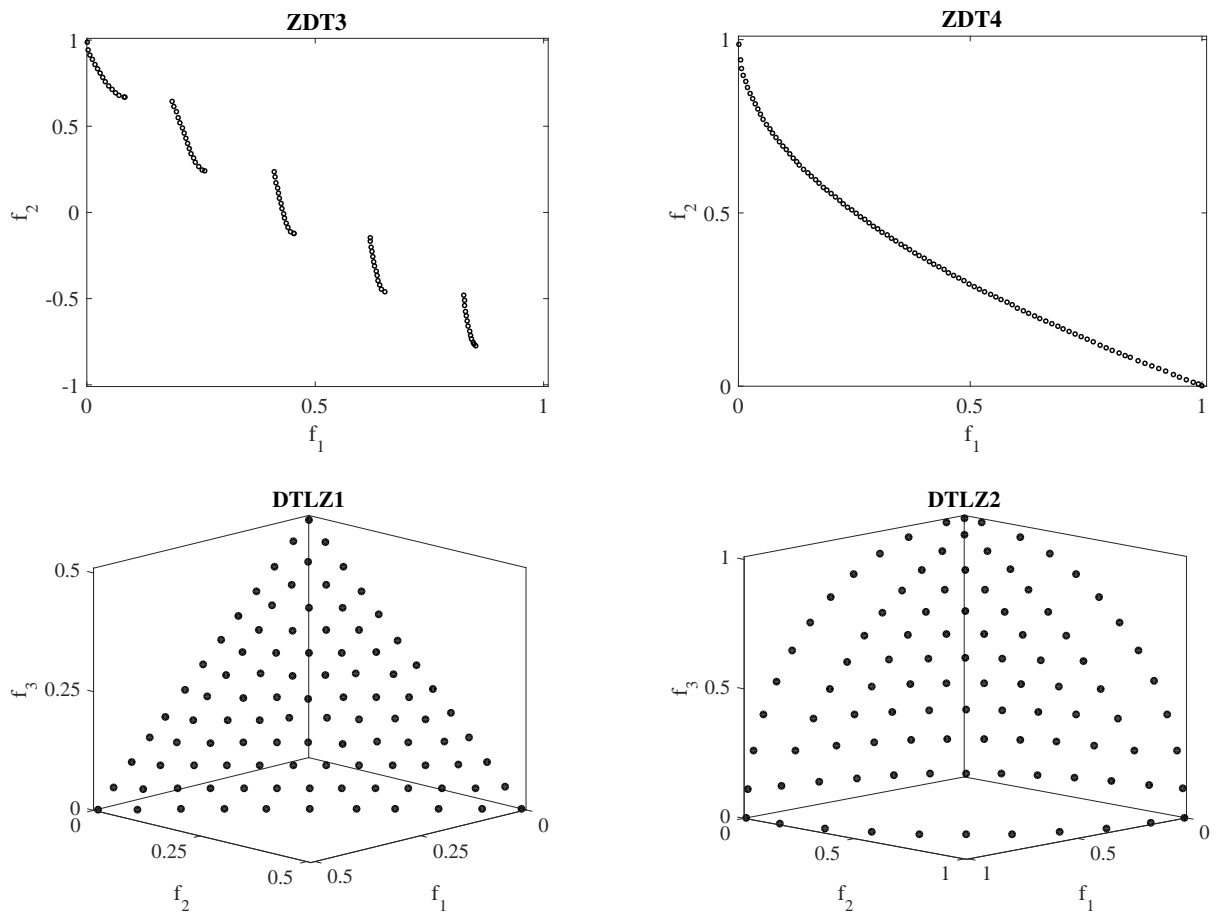

Fig. 4: Pareto front approximations obtained by MOEA/D-WSFM on some ZDT and DTLZ problems.

Table 2 shows the results in terms of the quality indicators obtained by different MOEA/D variants. It is apparent that WSFM produces highly competitive performance. When the global replacement strategy is used, WSFM is only outperformed on LZ09_F6. This is a three-objective problem with a spherical Pareto front. The ability to deal with such Pareto front geometry was demonstrated in the previous experiments. Eventually, a larger population size may be needed to successfully handle this problem [11]. Overall, the obtained results further confirm a trend that was observed so far. Specifically, the performance of WSFM becomes more likely superior to other methods when the replacement is performed in the neighborhood of the best matching subproblem to the offspring. The reason is due to competitive characteristics of WSFM for preference articulation. As shown in [6], WSFM can identify solutions better reflecting the decision maker preferences expressed by weights when comparing with traditional methods. As results of this study show, this is translated into a better correspondence between individuals and respective subproblems during the search, which can improve the MOEA/D performance.

Figure 5 shows the evolution of the epsilon indicator on three challenging problems from the LZ09 suite. The plots in this figure illustrate that the use of 
Table 2: Results for LZ09 problems. The values refer to the median and interquartile range of the epsilon (eps) and hypervolume (hv) indicators.

\begin{tabular}{|c|c|c|c|}
\hline M & $\mathrm{HB}$ & BI & $\mathrm{W}$ \\
\hline \multicolumn{4}{|c|}{ MOEA/D } \\
\hline \multirow{2}{*}{ 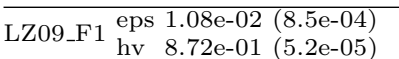 } & -03 (1.0e-04) & $2.83 \mathrm{e}-01(1.5$ & $1.80 \mathrm{e}-03(6$ \\
\hline & $-01(3.7 \mathrm{e}-05)$ & $41 \mathrm{e}-01(7.7$ & $8.75 \mathrm{e}-01($ \\
\hline \multirow{2}{*}{ 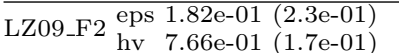 } & $1.18 \mathrm{e}-02(9.7 \mathrm{e}-03)$ & $3.46 \mathrm{e}-01(7.9 \mathrm{e}-02)$ & $7.21 \mathrm{e}-03(2.3 \mathrm{e}-03)$ \\
\hline & $8.69 \mathrm{e}-01(1.8 \mathrm{e}-03)$ & $6.55 \mathrm{e}-01(5.6 \mathrm{e}-02)$ & $8.71 \mathrm{e}-01(1.5 \mathrm{e}-03)$ \\
\hline \multirow{2}{*}{ 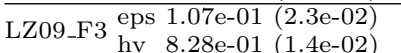 } & $1.57 \mathrm{e}-02(3.2 \mathrm{e}-02)$ & $4.25 \mathrm{e}-01(4.1 \mathrm{e}-02)$ & $9.25 \mathrm{e}-03(1.7 \mathrm{e}-02)$ \\
\hline & $8.70 \mathrm{e}-01(4.4 \mathrm{e}-03)$ & $6.54 \mathrm{e}-01(2.9 \mathrm{e}-02)$ & $8.71 \mathrm{e}-01(2.1 \mathrm{e}-03)$ \\
\hline \multirow{2}{*}{ LZ09_F4 eps $1.35 \mathrm{e}-01(3.0 \mathrm{e}-02)$} & $4.34 \mathrm{e}-02(5.1 \mathrm{e}-02)$ & $4.30 \mathrm{e}-01(5.2 \mathrm{e}-02)$ & $1.04 \mathrm{e}-02(1.3 \mathrm{e}-02)$ \\
\hline & $8.67 \mathrm{e}-01(9.9 \mathrm{e}-03)$ & $6.51 \mathrm{e}-01(3.6 \mathrm{e}-02)$ & $8.71 \mathrm{e}-01(3.1 \mathrm{e}-03)$ \\
\hline \multirow{2}{*}{$\begin{array}{lll}\text { LZ09_F5 } & \text { eps } 1.24 \mathrm{e}-01 & (9.6 \mathrm{e}-03) \\
\text { hv } & 8.12 \mathrm{e}-01 & (9.8 \mathrm{e}-03)\end{array}$} & $\mathrm{e}-02(3.1 \mathrm{e}-02)$ & $3.82 \mathrm{e}-01(4.7 \mathrm{e}-02)$ & $6.51 \mathrm{e}-02(2.6 \mathrm{e}-02)$ \\
\hline & $01(5.9 \mathrm{e}-03)$ & $-01(3.0$ & $8.60 \mathrm{e}-01(4$. \\
\hline \multirow{2}{*}{ 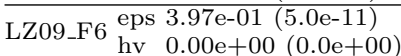 } & $1.34 \mathrm{e}-01(4.6 \mathrm{e}-02)$ & $5.02 \mathrm{e}-02(7.1 \mathrm{e}-03)$ & $1.08 \mathrm{e}-01(2.1 \mathrm{e}-02)$ \\
\hline & $7.07 \mathrm{e}-01(1.2 \mathrm{e}-02)$ & $7.63 \mathrm{e}-01(1.6 \mathrm{e}-03)$ & $7.34 \mathrm{e}-01(7.6 \mathrm{e}-03)$ \\
\hline \multirow{2}{*}{$\begin{array}{l}\text { LZ09_F7 eps } 2.12 \mathrm{e}-01(5.0 \mathrm{e}-02) \\
\text { hv } 7.84 \mathrm{e}-01(3.9 \mathrm{e}-02)\end{array}$} & $2.93 \mathrm{e}-03(4.0 \mathrm{e}-04)$ & $1.71 \mathrm{e}-01(1.8 \mathrm{e}-02)$ & $1.91 \mathrm{e}-03(1.2 \mathrm{e}-04)$ \\
\hline & $8.75 \mathrm{e}-01(8.5 \mathrm{e}-05)$ & $7.89 \mathrm{e}-01(7.8 \mathrm{e}-03)$ & $8.75 \mathrm{e}-01(7.0 \mathrm{e}-05)$ \\
\hline \multirow{2}{*}{$\begin{array}{l}\text { LZ09_F8 eps } 3.77 \mathrm{e}-01(5.4 \mathrm{e}-02) \\
\text { hv } 6.10 \mathrm{e}-01(7.5 \mathrm{e}-02)\end{array}$} & $\mathrm{e}-03(1.6 \mathrm{e}-03)$ & $3.62 \mathrm{e}-01(2.0 \mathrm{e}-01)$ & $2.48 \mathrm{e}-03(4.5 \mathrm{e}-04)$ \\
\hline & e-01 (7.4e-04) & $6.21 \mathrm{e}-01(9.5 \mathrm{e}$ & $8.74 \mathrm{e}-01(2.8 \mathrm{e}-04)$ \\
\hline \multirow{2}{*}{$\begin{array}{cl}\text { LZ09_F9 } \begin{array}{l}\text { eps } \\
\text { hv }\end{array} .81 \mathrm{e}-01(2.3 \mathrm{e}-07) \\
\end{array}$} & $-02(8.9 e-03)$ & $01(1.8$ & $02(1$. \\
\hline & $\mathrm{e}-01(4.1$ & $01(8.2$ & \\
\hline \multicolumn{4}{|c|}{ MOEA/D-GR } \\
\hline \multirow{2}{*}{ 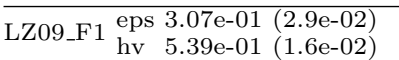 } & e-03 (6.1e-05) & $-01(1.2$ & 1.8 \\
\hline & $8.75 \mathrm{e}-01(3.6 \mathrm{e}-05)$ & $4 \mathrm{e}-01(6.9 \mathrm{c}$ & $01(2$ \\
\hline \multirow{2}{*}{ 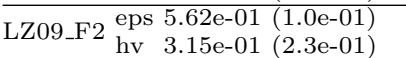 } & $1.07 \mathrm{e}-02(4.3 \mathrm{e}-03)$ & $3.31 \mathrm{e}-01(8.6 \mathrm{e}-02)$ & $6.00 \mathrm{e}-03(3.0 \mathrm{e}-03)$ \\
\hline & $8.70 \mathrm{e}-01(1.4 \mathrm{e}-03)$ & $6.65 \mathrm{e}-01(5.8 \mathrm{e}-02)$ & $8.71 \mathrm{e}-01(1.1 \mathrm{e}-03)$ \\
\hline \multirow{2}{*}{$\begin{array}{l}\text { LZ09_F3 eps } 5.33 \mathrm{e}-01(4.2 \mathrm{e}-02) \\
\text { hv } 2.83 \mathrm{e}-01(5.8 \mathrm{e}-02)\end{array}$} & $\mathrm{e}-02(1.5 \mathrm{e}-02)$ & $01(3$. & -03 \\
\hline & $01(2.2$ & $01(2$. & -01( \\
\hline \multirow{2}{*}{$\begin{array}{l}\text { LZ09_F4 eps } 5.35 \mathrm{e}-01(6.3 \mathrm{e}-02) \\
\text { hv } 2.94 \mathrm{e}-01\end{array}$} & $3.07 \mathrm{e}-02(2.8 \mathrm{e}-02)$ & $4.26 \mathrm{e}-01(4.8 \mathrm{e}-02)$ & $6.94 \mathrm{e}-03(2.4 \mathrm{e}-03)$ \\
\hline & $8.69 \mathrm{e}-01(2.9 \mathrm{e}-03)$ & $6.53 \mathrm{e}-01(3.4 \mathrm{e}-02)$ & $8.72 \mathrm{e}-01(8.5 \mathrm{e}-04)$ \\
\hline \multirow{2}{*}{$\begin{array}{lll}\text { LZ09_F5 } & \text { eps } 5.02 \mathrm{e}-01 & (4.5 \mathrm{e}-02) \\
\text { hv } & 3.27 \mathrm{e}-01 & (7.5 \mathrm{e}-02)\end{array}$} & $6.75 \mathrm{e}-02(2.9 \mathrm{e}-02)$ & $3.89 \mathrm{e}-01(3.0 \mathrm{e}-02)$ & $6.05 \mathrm{e}-02(3.0 \mathrm{e}-02)$ \\
\hline & $8.58 \mathrm{e}-01(6.1 \mathrm{e}-03)$ & $6.77 \mathrm{e}-01(2.0 \mathrm{e}-02)$ & $8.61 \mathrm{e}-01(4.6 \mathrm{e}-03)$ \\
\hline \multirow{2}{*}{ 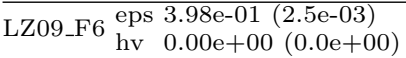 } & $1.25 \mathrm{e}-01(3.6 \mathrm{e}-02)$ & $6.70 \mathrm{e}-02(5.5 \mathrm{e}-03)$ & $1.05 \mathrm{e}-01(2.3 \mathrm{e}-02)$ \\
\hline & $7.10 \mathrm{e}-01(1.0 \mathrm{e}-02)$ & $7.50 \mathrm{e}-01(2.4 \mathrm{e}-03)$ & $7.39 \mathrm{e}-01(9.0 \mathrm{e}-03)$ \\
\hline \multirow{2}{*}{$\begin{array}{ccc}\text { LZ09_F7 } & \text { eps } 6.05 \mathrm{e}-01 & (1.4 \mathrm{e}-02) \\
\text { hv } & 2.33 \mathrm{e}-01 & (2.7 \mathrm{e}-02)\end{array}$} & $3.05 \mathrm{e}-03(3.9 \mathrm{e}-04)$ & $1.69 \mathrm{e}-01(3.1 \mathrm{e}-01)$ & $1.90 \mathrm{e}-03(1.4 \mathrm{e}-04)$ \\
\hline & $8.75 \mathrm{e}-01(7.5 \mathrm{e}-05)$ & $7.88 \mathrm{e}-01(1.8 \mathrm{e}-01)$ & $8.75 \mathrm{e}-01(8.6 \mathrm{e}-05)$ \\
\hline \multirow{2}{*}{$\begin{array}{l}\text { LZ09_F8 eps } 6.16 \mathrm{e}-01(4.0 \mathrm{e}-03) \\
\text { hv } 2.13 \mathrm{e}-01(8.1 \mathrm{e}-03)\end{array}$} & $4.06 \mathrm{e}-03(8.9 \mathrm{e}-03)$ & $5.01 \mathrm{e}-01(2.1 \mathrm{e}-01)$ & $2.65 \mathrm{e}-03(1.0 \mathrm{e}-03)$ \\
\hline & $8.74 \mathrm{e}-01(2.8 \mathrm{e}-03)$ & $5.96 \mathrm{e}-01(1.1 \mathrm{e}-01)$ & $8.74 \mathrm{e}-01(6.1 \mathrm{e}-04)$ \\
\hline LZ09 F9 eps 3.82e-01 (2.5e-02) & $1.56 \mathrm{e}-02(1.2 \mathrm{e}-02)$ & $1.06 \mathrm{e}-01(7.8 \mathrm{e}-02)$ & $1.43 \mathrm{e}-02(1.1 \mathrm{e}-02)$ \\
\hline LZ09_F9 hv $0.00 \mathrm{e}+00(0.0 \mathrm{e}+0$ & $5.34 \mathrm{e}-01(2.2 \mathrm{e}-03)$ & $4.94 \mathrm{e}-01(4.0 \mathrm{e}-02)$ & $5.35 \mathrm{e}-01(2.5 \mathrm{e}-03)$ \\
\hline
\end{tabular}
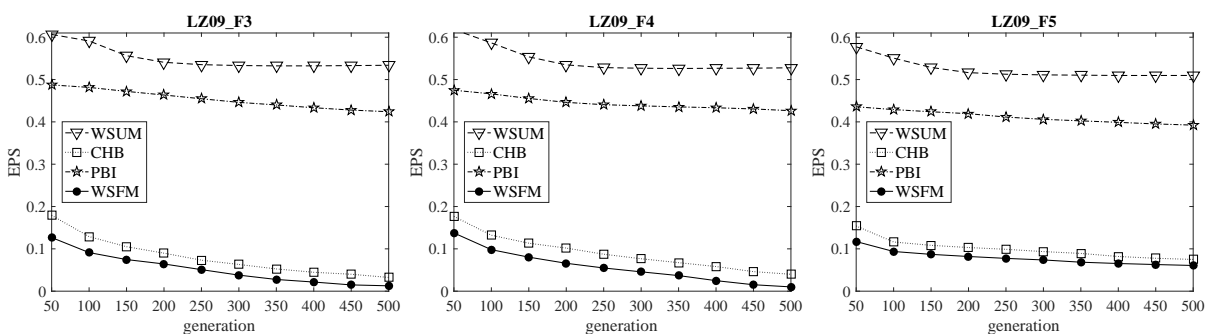

Fig. 5: Evolution of the epsilon indicator. The plots refer to the mean values over 30 runs. The lower the better. 
WSFM not only allows obtaining approximation sets with better values of the quality indicators but also can provide a faster convergence during the generations. This feature is especially useful when handling real-world problems where function evaluations can be computationally expensive.

\subsection{Engineering Problems}

Analyzing the performance of different MOEAs on artificially constructed test problems is advantageous, as the Pareto sets and the properties of these problems are known. However, such problems often do not pose difficulties that are encountered in real-world applications, being frequently criticized due to this fact. To illustrate a practical relevance and validity of MOEA/D-WSFM, three engineering problems are selected from the literature for experiments. Two considered problems refer to the design of four bar truss [15] and welded beam [3]. Both problems involve two objectives and four design variables that are restricted by box constraints. In addition, four inequality constraints are associated with the design of welded beam. The third is car side impact problem [8], which involves three objectives and seven variables as well as ten inequality constraints.

Table 3 presents the results of the application of different MOEA/D variants to solving engineering problems. These results suggest that MOEA/D-WSFM performs the best on these problems regarding the quality indicators. This is valid for both replacement strategies.

Figure 6 displays the approximations to the Pareto fronts with the best hypervolume values obtained with WSFM. The presented plots show that WSFM can effectively approximate the Pareto fronts for engineering problems. The obtained results demonstrate that WSFM not only exhibits a competitive performance on some test problem but also can be useful in practical applications. Also, WSFM can be successfully combined with a penalty function for handling constraints.

Table 3: Results for engineering problems. The values refer to the median and interquartile range of the epsilon (eps) and hypervolume (hv) indicators.

\begin{tabular}{|c|c|c|c|c|}
\hline & WSUM & $\mathrm{CHB}$ & PBI & WSFM \\
\hline \multicolumn{5}{|c|}{ MOEA/D } \\
\hline \multirow{2}{*}{ Four Bar Truss } & eps $1.17 \mathrm{e}-02(1.0 \mathrm{e}-03)$ & $7.20 \mathrm{e}-03(3.2 \mathrm{e}-05)$ & $2.39 \mathrm{e}-01(3.7 \mathrm{e}-02)$ & $4.88 \mathrm{e}-03(9.8 \mathrm{e}-05)$ \\
\hline & hv $6.71 \mathrm{e}-01(2.7 \mathrm{e}-05)$ & $6.74 \mathrm{e}-01(3.9 \mathrm{e}-05)$ & $5.62 \mathrm{e}-01(1.8 \mathrm{e}-02)$ & $6.74 \mathrm{e}-01(1.3 \mathrm{e}-05)$ \\
\hline \multirow{2}{*}{ Welded Beam } & eps $4.34 \mathrm{e}-03(3.0 \mathrm{e}-03)$ & $4.91 \mathrm{e}-03(2.8 \mathrm{e}-03)$ & $4.53 \mathrm{e}-02(1.9 \mathrm{e}-02)$ & $3.44 \mathrm{e}-03(3.5 \mathrm{e}-03)$ \\
\hline & hv $9.93 \mathrm{e}-01(2.6 \mathrm{e}-03)$ & $9.92 \mathrm{e}-01(2.6 \mathrm{e}-03)$ & $9.49 \mathrm{e}-01(1.5 \mathrm{e}-02)$ & $9.94 \mathrm{e}-01(3.0 \mathrm{e}-03)$ \\
\hline \multirow{2}{*}{ Car Side Impact } & eps $5.17 \mathrm{e}-01(0.0 \mathrm{e}+00)$ & $1.91 \mathrm{e}-01(7.3 \mathrm{e}-02)$ & $9.99 \mathrm{e}-01(3.6 \mathrm{e}-03)$ & $1.76 \mathrm{e}-01(3.0 \mathrm{e}-02)$ \\
\hline & hv $2.68 \mathrm{e}-01(3.9 \mathrm{e}-16)$ & $4.74 \mathrm{e}-01(1.5 \mathrm{e}-02)$ & $8.14 \mathrm{e}-04(3.6 \mathrm{e}-03)$ & $4.85 \mathrm{e}-01(8.5 \mathrm{e}-03)$ \\
\hline \multicolumn{5}{|c|}{ MOEA/D-GR } \\
\hline \multirow{2}{*}{ Four Bar Truss } & eps $1.74 \mathrm{e}-01(3.6 \mathrm{e}-02)$ & $7.19 \mathrm{e}-03(2.7 \mathrm{e}-05)$ & $2.47 \mathrm{e}-01(3.7 \mathrm{e}-02)$ & $\overline{4.85 \mathrm{e}-03(8.2 \mathrm{e}-05)}$ \\
\hline & hv $5.02 \mathrm{e}-01(3.0 \mathrm{e}-02)$ & $6.74 \mathrm{e}-01(3.7 \mathrm{e}-05)$ & $5.60 \mathrm{e}-01(1.4 \mathrm{e}-02)$ & $6.74 \mathrm{e}-01(2.5 \mathrm{e}-05)$ \\
\hline \multirow{2}{*}{ Welded Beam } & eps $4.08 \mathrm{e}-02(1.5 \mathrm{e}-02)$ & $4.79 \mathrm{e}-03(2.8 \mathrm{e}-03)$ & $7.60 \mathrm{e}-02(3.5 \mathrm{e}-02)$ & $2.81 \mathrm{e}-03(2.2 \mathrm{e}-03)$ \\
\hline & hv $9.85 \mathrm{e}-01(1.5 \mathrm{e}-02)$ & $9.93 \mathrm{e}-01(1.7 \mathrm{e}-03)$ & $9.23 \mathrm{e}-01(3.5 \mathrm{e}-02)$ & $9.95 \mathrm{e}-01(2.8 \mathrm{e}-03)$ \\
\hline \multirow{2}{*}{ Car Side Impact } & eps $3.42 \mathrm{e}-01(7.6 \mathrm{e}-02)$ & $1.74 \mathrm{e}-01(4.5 \mathrm{e}-02)$ & $4.08 \mathrm{e}-01(1.8 \mathrm{e}-01)$ & $1.74 \mathrm{e}-01(1.5 \mathrm{e}-02)$ \\
\hline & hv $1.93 \mathrm{e}-01(5.1 \mathrm{e}-02)$ & $4.76 \mathrm{e}-01(9.1 \mathrm{e}-03)$ & $3.33 \mathrm{e}-01(1.5 \mathrm{e}-01)$ & $4.89 \mathrm{e}-01(9.2 \mathrm{e}-03)$ \\
\hline
\end{tabular}



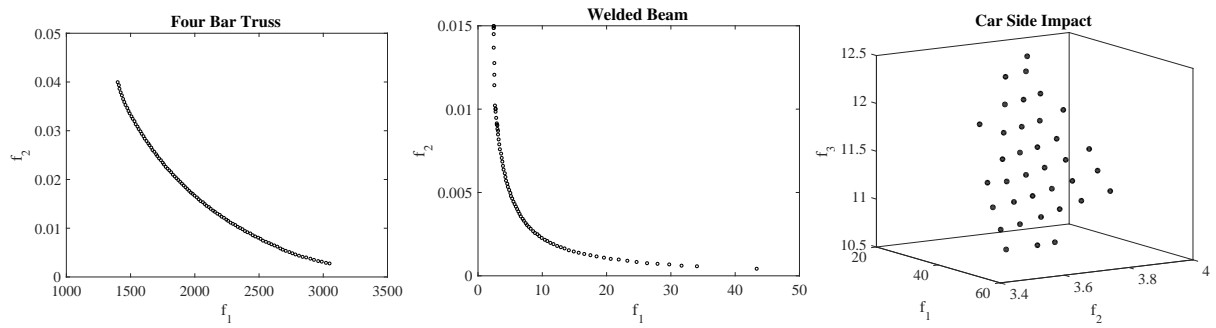

Fig. 6: Pareto fronts obtained by MOEA/D-WSFM for engineering problems.

\section{Conclusions}

MOEA/D is a popular state-of-the-art framework in the field of evolutionary multiobjective optimization. By means of scalarization, MOEA/D decomposes a MOP into a number of single-objective subproblems. During the run, subproblems are optimized simultaneously exploiting the neighborhood relations between them. The scalarizing function is an important issue that largely affects the performance of MOEA/D.

This study suggested a new scalarizing function for MOEA/D, named WSFM. It is characterized by the stress function that performs a nonlinear mapping taking into account objective and weight values. This process is inspired by the stress-strain behavior of thermoplastic vulcanizates. The experimental results suggest that MOEA/D with WSFM can achieve a faster convergence and better final results with respect to quality indicators. WSFM works particularly better when the global replacement strategy is used. The validity of the approach is also demonstrated on some engineering problems, highlighting its practical relevance.

As future work, there are plans to investigate the performance of WSFM on other test and real-world problems, including those having a large number of objectives. Also, the development of an adaptive scheme for controlling the parameters in WSFM is a promising research direction.

Acknowledgements This work has been supported by FCT - Fundação para a Ciência e Tecnologia in the scope of the project: PEst-OE/EEI/UI0319/2014.

\section{References}

1. Abdou-Sabet, S., Datta, S.: Thermoplastic vulcanizates. Polymer Blends: Formulation and Performance, Wiley, New York, US (2000)

2. Deb, K., Pratap, A., Agarwal, S., Meyarivan, T.: A fast and elitist multiobjective genetic algorithm: NSGA-II. IEEE Trans. on Evol. Comput. 6(2), 182-197 (2002)

3. Deb, K., Pratap, A., Moitra, S.: Mechanical component design for multiple ojectives using elitist non-dominated sorting ga. In: Schoenauer, M., Deb, K., Rudolph, G., Yao, X., Lutton, E., Merelo, J.J., Schwefel, H.P. (eds.) PPSN VI, LNCS, vol. 1917, pp. 859-868. Springer Berlin Heidelberg (2000) 
4. Denysiuk, R., Costa, L., Espírito Santo, I.: MOEA/VAN: Multiobjective evolutionary algorithm based on vector angle neighborhood. In: Proc. Conf. Genet. Evol. Comput. pp. 663-670. (2015)

5. Denysiuk, R., Costa, L., Espírito Santo, I., Matos, J.C.: MOEA/PC: Multiobjective evolutionary algorithm based on polar coordinates. In: Gaspar-Cunha, A., Antunes, C.H., Coello, C.C. (eds.) EMO, LNCS, vol. 9018, pp. 141-155. Springer International Publishing (2015)

6. Ferreira, J.C., Fonseca, C.M., Gaspar-Cunha, A.: Methodology to select solutions from the Pareto-optimal set: A comparative study. In: Proc. Conf. Genet. Evol. Comput. pp. 789-796. (2007)

7. Ishibuchi, H., Sakane, Y., Tsukamoto, N., Nojima, Y.: Simultaneous use of different scalarizing functions in MOEA/D. In: Proc. Conf. Genet. Evol. Comput. pp. 519526. (2010)

8. Jain, H., Deb, K.: An evolutionary many-objective optimization algorithm using reference-point-based nondominated sorting approach, Part II: Handling constraints and extending to an adaptive approach. IEEE Trans. on Evol. Comput. 18(4), 577-601 (2002)

9. Jan, M.A., Zhang, Q.: MOEA/D for constrained multiobjective optimization: Some preliminary experimental results. In: UK Workshop on Comput. Intell. pp. 1-6 (2010)

10. Knowles, J., Thiele, L., Zitzler, E.: A tutorial on the performance assessment of stochastic multiobjective optimizers. Tech. Rep. 214, TIC, ETH Zurich, Switzerland (2006)

11. Li, H., Zhang, Q.: Multiobjective optimization problems with complicated Pareto sets, MOEA/D and NSGA-II. IEEE Trans. on Evol. Comput. 13(2), 284-302 (2009)

12. Li, K., Deb, K., Zhang, Q., Kwong, S.: An evolutionary many-objective optimization algorithm based on dominance and decomposition. IEEE Trans. on Evol. Comput. 19(5), 694-716 (2015)

13. Liu, H.L., Gu, F., Zhang, Q.: Decomposition of a multiobjective optimization problem into a number of simple multiobjective subproblems. IEEE Trans. on Evol. Comput. 18(3), $450-455$ (2014)

14. Miettinen, K.: Nonlinear multiobjective optimization. Kluwer Academic Publishers (1999)

15. Ray, T., Liew, K.M.: A swarm metaphor for multiobjective design optimization. Eng. Optim. 34(2), 141-153 (2002)

16. Wang, Z., Zhang, Q., Zhou, A., Gong, M., Jiao, L.: Adaptive replacement strategies for MOEA/D. IEEE Trans. Cybern. 46(2), 474-486 (2016)

17. Zhang, Q., Li, H.: MOEA/D: A multiobjective evolutionary algorithm based on decomposition. IEEE Trans. on Evol. Comput. 11(6), 712-731 (2007)

18. Zhou, A., Zhang, Q.: Are all the subproblems equally important? Resource allocation in decomposition-based multiobjective evolutionary algorithms. IEEE Trans. on Evol. Comput. 20(1), 52-64 (2016)

19. Zitzler, E., Künzli, S.: Indicator-Based Selection in Multiobjective Search. In: X. Yao, E. K. Burke, J. A. Lozano, J. Smith, J. J. Merelo-Guervós, J. A. Bullinaria, J. E. Rowe, P. Tiňo, A. Kabán, H.-P. Schwefel (eds.) PPSN VIII, LNCS, vol. 3242, pp. 832-842. Springer Berlin Heidelberg (2004) 